\title{
Visualization of a Juvenile Australopithecus afarensis Specimen: Implications for Functional Foot Anatomy
}

\author{
Eleanor Milman, John Daugherty, Zeresenay Alemseged, Kevin Brennan, Leah Lebowicz
}

Since it was named in 1978, analyses of Australopithecus afarensis have culminated in several dominant theories on how humans acquired many of their unique adaptations. Because bipedal locomotion is one of the earliest characteristics of human functional anatomy to appear in the fossil record, its associated anatomy in early hominins has significant implications for human evolution (Stern 2000). The skeleton and overall morphological characteristics of the foot in Australopithecus afarensis provide important clues about the origins of upright bipedal locomotion. Popularly known as "Selam," the 3.3 million-year-old DIK-1-1 fossil was discovered in Dikika, Ethiopia by Dr. Zeresenay Alemseged and his team in 2000. Selam was an australopithecine who died at three years old, making her the youngest early hominin specimen known today (Alemseged et al. 2006). This discovery allows researchers to investigate not only locomotor patterns of A. afarensis within the context of human evolution, but also to examine what child development may have looked like during this pivotal time. The purpose of this project is to create a $3 D$ animation that accurately reconstructs the anatomy and taphonomy of the Dikika foot. By segmenting CT data, 3D modelling, and animating, this investigation aims to contribute to the breadth of fossil reconstruction techniques in the field of biomedical visualization. This method provides a robust means of communication within, and beyond, the paleoanthropological community about new discoveries and how to visualize them.

OPEN ACCESS

\section{Keywords}

Australopithecus afarensis, paleoanthropology, 3D animation, human evolution, segmentation, fossil

\section{Introduction}

\section{Overview of the Research Problem}

Biomedical visualization technology and methods have grown with, and influenced, the field of paleoanthropology for generations. In recent decades, computerized tomography and segmentation software have become some of the most popular tools for visualizing and analyzing fossil hominins (Spoor et al. 2000). From a stack of two-dimensional (2D) images (slices), three-dimensional (3D) models are built, analyzed, and animated. This allows (a) non-invasive exploration of complex functional morphologies, (b) virtual curation of rare fossils, and (c) improved accessibility of paleoanthropological research to enhance public education at museums and elsewhere (Spoor et al. 2000).

One of the best documented hominin species in the human fossil record is Australopithecus afarensis and includes some of the most iconic fossils such as Lucy and Selam. Since it was named in 1978, analyses of this species have culminated in several dominant theories on the evolution of how humans acquired many of their unique traits and related adaptations. Among these adaptations, the development of bipedal locomotion and the extent to which A. afarensis employed it, is the topic of a weighted discussion (Stern 2000). Because this form of locomotion is one of the earliest unique characteristics of human functional anatomy to appear in the fossil record, bipedalism and its associated anatomy in early hominins have significant implications for uncovering the story of human evolution (Stern 2000).

The skeletal morphology of the foot is naturally an important anatomical feature specialized for any species' locomotor adaptation. This investigation visualizes and animates the functional foot anatomy of a 3.3 million-year-old $A$. afarensis specimen (DIK-1-1). Popularly known as "Selam" or "Dikika Child," this fossil is unique among others of its species, because it belonged to a three-year-old juvenile individual and is almost complete. These properties allow researchers to explore beyond what is known about adult morphology and look at the growth patterns exhibited by these early australopithecines.

\section{Significance of the Problem}

The premise that hominins needed to move from the trees to the ground before developing many uniquely human characteristics is a common presumption among paleoanthropologists (Kimbel and Delezene 2009). Large brains, social complexity, and slow child development are generally believed to have evolved after bipedal locomotion, making this evolutionary milestone a topic of pivotal and scrupulous research (Kimbel and Delezene 2009). Throughout the last several decades, fossils have provided mixed evidence about the extent to which early hominins depended on bipedal locomotion (Harcourt-Smith and Aiello 2004).

Today, over $400 \mathrm{~A}$. afarensis specimens have been collected from six sites in eastern Africa that span from 3.7 to 3.0 million years ago (Kimbel and Delezene 2009). The discovery of $A$. afarensis in the 1970s marked a significant turning point in the study of human evolution, especially regarding expectations about the hominid divergence from African apes, bipedalism, ontogenetic growth patterns, and diet (Stern 
2000). The most well-known and complete fossil specimen from this species, commonly known as "Lucy," was discovered in Hadar, Ethiopia by Donald Johanson, Tom Gray, and their team of excavators in 1974 (Stern 2000). To this day, A. afarensis is generally understood (albeit with some contention) to be one of the earliest hominids capable of bipedal locomotion (Stern 2000). Since the discovery of Lucy, a rigorous debate over the precise origins and development of bipedalism has ensued.

\section{Literature Review}

\section{Functional Anatomy of the Modern Human Foot}

Before delving into a detailed comparison between humans and other apes, it is important to understand the general anatomy of the human foot bones and their function in bipedal locomotion.

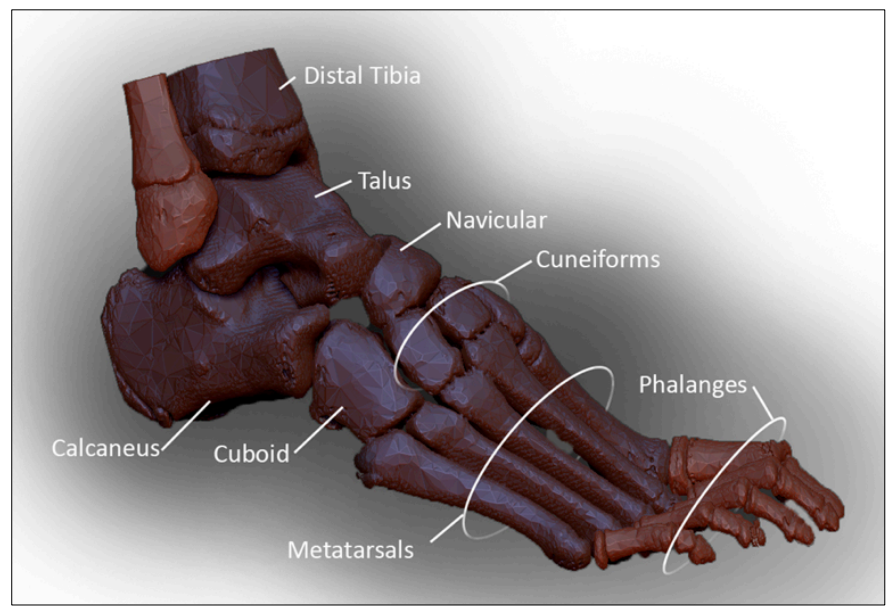

Figure 1. 3D visualization from CT data of modern human 11-year old foot skeleton with bones indicated.

Several key features of the modern human foot distinguish it from that of Pan troglodytes (chimpanzees) and other extant great apes. These include the presence of a mediallongitudinal arch, an adducted hallux, and reduced pedal phalanges (Kanamoto et al. 2011).

In 2015, a study was conducted to analyze the opposability of the hallux in humans and great apes of different developmental stages (Gill et al. 2015). The researchers examined the medial cuneiform bone, which can provide insight on the opposability of the big toe as it articulates with the first metatarsal bone, the hallux; and the navicular. It contributes to the transverse arch of the foot and serves as an attachment site for several muscles that invert and evert the foot. In great apes, these articulations are essential for abducting and grasping during arboreal locomotion; in humans, they play a key role in stabilization and propulsion during bipedal locomotion (Gill et al. 2015). Thus, the medial cuneiform and its precise shape have significant implications for the basis of locomotor differences between living hominoids. Of special relevance to the current project is the study's investigation into the ontogenetic development of the medial cuneiform. Modern human children have a medial cuneiform with a convex and medially-oriented surface on its distal facet, which flattens and becomes more distally-oriented with age (Gill et al. 2015). Young chimpanzees display a similar growth pattern but to a significantly lesser extent (Gill et al. 2015). The authors note that since skeletal growth is responsive to the mechanical environment, the developing medial cuneiform bone should theoretically grow to reflect the type of locomotor pattern adopted by the individual.

Similar research has also examined the shape of the talus and its articular surfaces. In 2010, Kanamoto et al. examined the shape of the talus in humans and other great apes, including Pan troglodytes (chimpanzees). Because of its position in the foot, the shape and articulations of the talus dictate the overall function of the surrounding structures. In humans, the superior articular surface is more parallel with the horizontal plane of the talar body than it is in apes; the cylindrical axis is directed less anteroposteriorly; the subtalar axis is more anteroposteriorly oriented; and the "navicular articular surface is more plantarly-oriented and axially-twisted," (Kanamoto et al. 2010, p. 67). The authors note that "many comparative studies of the primate talus have been conducted to reconstruct the locomotion of fossil hominids and understand the evolution of human bipedalism" (p. 62). This perspective has helped to establish how morphological differences in humans reflect adaptations to bipedal locomotion, while the anatomy of apes is specialized for some arborealism.

\section{The Evolution of Bipedal Locomotion}

Recent 3D analyses of foot bones suggest greater diversity in human bipedalism than originally thought, especially considering the intermediate body proportions of $A$. afarensis compared to Homo habilis and modern humans. The authors of this study write that, "although perhaps controversial, it is important that when considering such a unique adaptation as bipedalism, we do not allow that uniqueness to imply that there was ever only one successful mode of bipedalism in our hominin ancestry," (Harcourt-Smith and Aiello 2004, p. 413).

At present, the debate over locomotion in A. afarensis falls into two positions:

1. A. afarensis was an obligate biped with a striding gait. This view focuses on features of the A. afarensis skeleton that resemble that of modern humans, a.k.a. derived characteristics.

2. A. afarensis retained at least some arboreal behavior from its ancestors, and if it was bipedal, it was in a manner different from humans. This view focuses on features of the skeleton that resemble modern apes, a.k.a. primitive characteristics.

Among these views are, "surprisingly few disagreements about fossil morphology;" the debate is instead over, "how to reconstruct function from morphology and relate function to behavior," (Kimbel and Delezene 2009, p. 36). Due to the limited fossil record of earlier species, a resolution to this 
debate remains elusive. Partially as both a consequence and a cause of this limitation, one of the major setbacks, thus far, is the consistent reliance on a, "dichotomous chimpanzee-human comparative framework," (Kimbel and Delezene 2009, p. 39). DeSilva (2009) introduces an important note of caution about using a chimpanzee model for studying the locomotion of early hominins. After observing a group of chimpanzees for a study, he notes that if early hominins climbed trees, then they did it differently from chimpanzees. Kimbel and Delezene (2009) write that, " $A$. afarensis was in no evolutionary sense intermediate between chimpanzees and humans and there are simply no reasonable analogs to human striding bipedality elsewhere in the animal kingdom from which to draw lessons as to cause," (p. 39). This presents a crucial limitation in kinetic and morphological comparisons between the locomotive patterns of humans and any ancestral species.

According to Duncan, Kappelman, and Shapiro (1994), "every species is composed of characteristics that reflect both its ancestry as well as its unique evolutionary pathway; understanding the overall functional pattern of the organism requires an equal consideration of all its anatomical features," (p. 79). Considering this, it is important to appreciate that $A$. afarensis likely possessed vestigial traits of its ancestors much like modern humans do today.

\section{Selam (DIK-1-1)}

Popularly known as "Selam," the DIK-1-1 fossil was discovered in Dikika, Ethiopia by Dr. Zeray Alemseged and his team in 2000. With the help of stratigraphic scaling and radiometric dating, it was determined that the individual lived between 3.35 and 3.31 million years ago (Alemseged et al. 2006). Through analysis of her dentition, it is believed that Selam was about three years old when she died, making her the youngest early hominin specimen known today (Alemseged et al. 2006). This allows researchers to investigate not only locomotor patters of $A$. afarensis within the context of human evolution, but also to examine what child development may have looked like three million years ago. The significance of the fossil has been, "unmatched in the early hominin fossil record not only because of its completeness, but because it reveals previously unknown morphology and provides a comprehensive cranial and postcranial record of a juvenile australopith," (Alemseged et al. 2006, p. 299).

DIK-1-1 is potentially one of the most important contributions to the future of research on bipedal locomotion. While the postcranial skeleton has clarified several aspects of the bipedalism debate, it has obscured many others. In general, "the foot and the lower limb provide clear evidence for bipedal locomotion, but the gorilla-like scapula and long and curved manual phalanges raise new questions about the importance of arboreal behaviour in the A. afarensis locomotor repertoire," (Alemseged et al. 2006, p. 296). After several analyses, researchers believe that Selam's morphology displays something of a "functional dichotomy" in which the lower body is adapted largely for bipedal locomotion like modern humans, and the upper body is adapted largely for climbing, like apes (Alemseged et al. 2006, p. 300). Thus, most of the evidence for bipedalism in this specimen lies in the lower limb and foot.

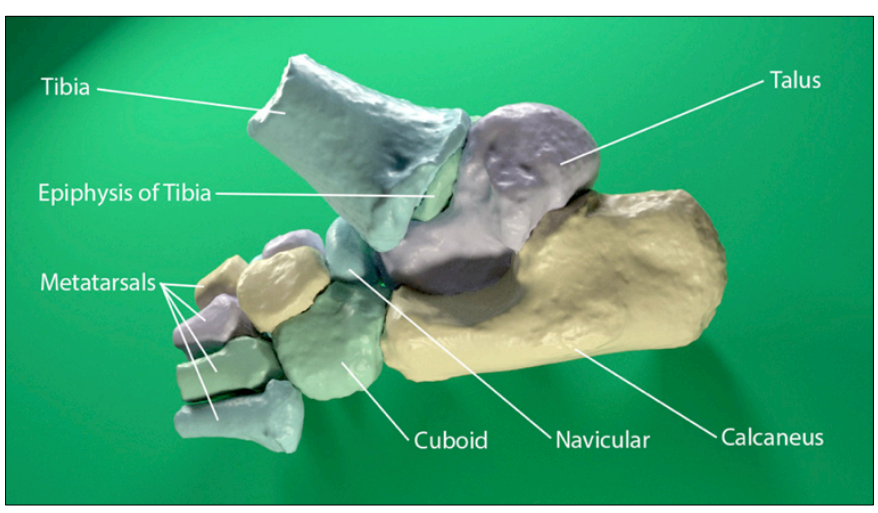

Figure 2. 3D render of segmented CT data from Dikika foot fossil.

\section{Visualization Techniques in Paleoanthropology}

Using 3D segmentation techniques, it is possible to generate a virtual 3D model of a fossil and visualize the complex spatial relationships between different structures. In the late 90s, 3D imaging was introduced as a means for visualizing the fossils of human ancestry (Spoor et al. 1999).

With improved scanners and segmentation software, it has become more feasible in recent years to digitally remove the sandstone matrix that fills fossil cavities, so that researchers may examine the contours of the fossilized bone itself. Researchers have since overcome many of the previous limitations by employing automated segmentation techniques such as multiplanar reformatting, interpolation, and volume rendering (Spoor et al. 1999). A 2014 review paper by Cunningham and colleagues suggests that, in comparison to earlier techniques for fossil analysis and reconstruction, 3D imaging allows for more objectivity, and has thus "overhauled and revitalized" comparative anatomical studies of extinct species (Cunningham et al. 2014, p. 352).

Substantial innovation in 3D fossil visualization and reconstruction has taken place over the last decade, especially with collaboration between the Biomedical Visualization Graduate Program at the University of Illinois at Chicago (UIC) and Dr. Paul Sereno in the Department of Organismal Biology and Anatomy at the University of Chicago. In 2011, Melissa Sisk of UIC used modern digital sculpting and painting techniques to reconstruct the face of a male specimen from the early Holocene (Sisk 2011). In 2012, Katherine Henning used 3D animation techniques to reconstruct the stature of a mid-Holocene male (Henning 2012). In 2015, Alejandro Padilla used Materialise Mimics software to build 3D models and develop an interactive PDF from CT images of human paleontological remains from the Gobero burial site (Padilla 2015) The Gobero archaeological site, dating to 
approximately $8000 \mathrm{BCE}$, is the oldest known graveyard in the Sahara Desert. In 2017, Katherine Zumach digitally reconstructed and created a 3D animation of a Norellius nyctisaurops specimen, while also developing a methodology for the overall procedure (Zumach 2017). Together, these projects, and others like them, have pioneered methods of visualizing fossilized remains of extinct species. Through segmentation, researchers can make spatial evaluations and hypotheses; through modeling and painting, they can understand specimens in a more lifelike context; and through $3 \mathrm{D}$ animation, they can view specimens as multidimensional living creatures.

Paleontological visualization specialists Boucher and Artz (2013) highlight that accurate reconstructions, such as paleoart, enhance the accessibility of fossil discoveries to the point that, "scientists are now using new forms of paleoimagery in order to test their own hypotheses," (p. 15). Visualizations have become a means for researchers not only to illustrate existing information, but also to make novel discoveries and gain new insights.

3D animation allows researchers to view their hypotheses of how early hominins moved about their environment in a way that brings them to life (Henning 2012). This may provide a means by which researchers can analyze and differentiate hypotheses on the evolution of bipedal locomotion and may generate new theories based on visualizations of existing ones. This idea was explored in a 2014 study that examined the role of animations in the mental representation of $3 \mathrm{D}$ objects. Münzer found that when participants studied static images, they were more likely to answer aptitude questions correctly if they had a prior high spatial ability. When participants viewed animations before testing, however, this effect was reduced and the scores of those with lower spatial abilities improved drastically (Münzer 2014). This work suggests that animation allows those with poorer spatial ability to comprehend spatial information more successfully. Further, it also reveals the value of animation in the global communication of visual information.

\section{Research Gap}

Some of the challenges scientists face in uncovering the functional morphology and locomotor pattern of $A$. afarensis include:

1. In any investigation of an extinct species, living analogs provide essential insight; as a result, modern humans and chimpanzees have become the main proxy for comparisons that involve fossil hominins. This often leads to the tempting, but problematic, assumption that australopithecines were an intermediate between "primitive" chimpanzees and "sophisticated" humans (Kimbel and Delezene 2009).

2. A limited fossil record of earlier species prevents researchers from determining which features of $A$. afarensis were derived, and which were ancestral. Because the last common ancestor between Pan troglodytes (chimpanzees) and Homo sapiens is still unknown, it is difficult to clarify whether $A$. afarensis retained its features from this ancestor, or if the traits evolved separately (Green and Alemseged 2012).

3. Most of the current research on A. afarensis is derived from fossils that belonged to adult individuals. Without juvenile specimens, research or analysis about their life histories, growth, or ontogenetic development is sparse.

4. Various hypotheses addressing the locomotion of $A$. afarensis rest on the interpretations of locomotor function from skeletal morphology, and when said skeletal morphology is only available in the form of fossils, visualization plays a significant role in researchers' various interpretations (Padilla 2015).

This investigation addresses challenges 3 and 4 .

\section{Research Significance}

\section{Significance of Research Study}

The general lack of infant and juvenile fossils has become a severe limitation in the ability of researchers to make inferences about the growth patterns and child development of early human ancestors. Because infant and juvenile skeletal remains are more fragile than that of adults, they are less likely to preserve well, and as a result, discovery of their remains are far less common. The 3.3 million years ago (mya) Dikika fossil has been described as a pivotal discovery in paleoanthropology, representing some of the oldest remains of an early hominin child.

Even with juvenile additions to the fossil record, inferring function from morphology requires some form of visual reconstruction. The development and application of accurate, high-quality reconstruction techniques, such as $3 \mathrm{D}$ modeling and animation, help clarify differing interpretations of the fossil record. 3D animation is an especially useful tool in evaluating hypotheses that pertain to functional anatomy because of its ability to clarify and elucidate verbal analyses of morphology, and to depict complex spatial and temporal information in $3 \mathrm{D}$ space.

\section{Research Question}

How can an animation be created to visualize the taphonomy and functional foot anatomy of a juvenile $A$. afarensis specimen; and enable further investigation of mechanical function, facilitate public outreach, and contribute to the study of human evolution?

\section{Methods}

\section{Research Study Design}

The purpose of this project was to create a $3 \mathrm{D}$ animation that accurately reconstructs the anatomy and taphonomy of the Dikika foot. The study design utilized existing segmentation 
and animation technology with a novel workflow and subject matter. The segmentation process ensured that accurate visualizations were being collected. The segmented CT data were converted into 3D models, which were animated and composited. This 3D visualization of the Dikika fossil was supplemented with CT data from a pediatric human foot for comparison. Footage from the Dikika excavation site was also incorporated into the animation.

\section{Stimulus Design Plan \\ Preproduction}

Segmentation: The physical DIK-1-1 fossil remained in Ethiopia, where it was scanned using Computerized Tomography (CT). The data were stored as a stack of approximately 1400 two-dimensional TIFF images with a voxel size of $0.03 \mathrm{~mm}$. This stack of images was sent to Dr. Alemseged's laboratory at the University of Chicago. Here, the images were accessed using Avizo Lite materials science software. First, the skeleton of the lower limb was separated from the rest of the fossil. Lower limb bones included the distal tibia, calcaneus, talus, cuboid, navicular, metatarsals V, IV, III, II, and the base of I, the medial, intermediate, and lateral cuneiform bones, and the epiphyseal growth plate of the distal tibia (Figure 2).

To segment the images, the fossilized bone was digitally separated from the surrounding rock matrix. Because of the relative similarity in density between these two materials, this phase could not be automated, and each image slice was segmented by hand removing the non-osseous material. Next, the foot bones were separated from each other. Portions of each slice that belonged to the target bone were segmented using a Wacom art tablet. In the interest of practicality, only one of every three slices were segmented. This reduced the resolution by a factor of three but did not significantly impact the amount of detail preserved in the reconstruction. Each of the thirteen foot bones (plus the epiphyseal growth plate at the distal tibia) was given its own virtual material. These materials were reformatted into DICOM data sets. One DICOM set was exported for each of the 14 foot structures.

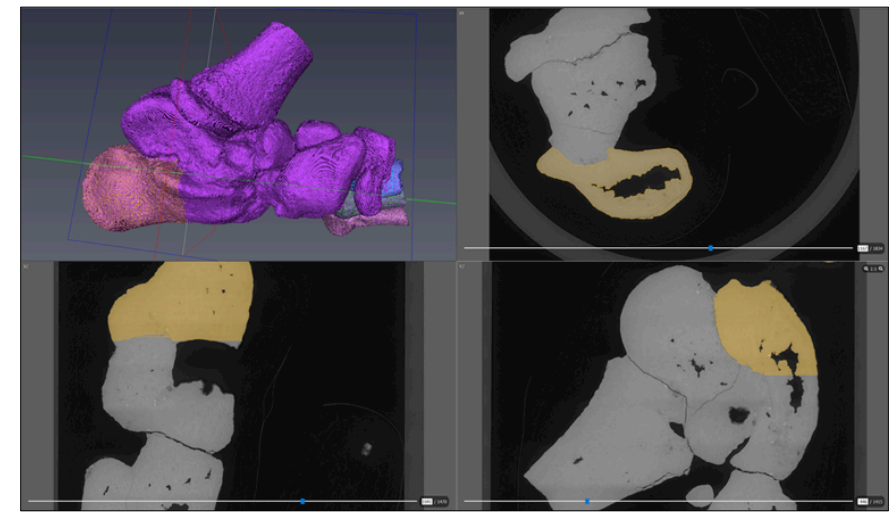

Figure 3. Avizo segmentation interface with 3D, coronal, transverse, and sagittal views of Dikika fossil foot depicted. The calcaneus is in the process of segmentation.
Model Creation: The fourteen DICOM sets created in Avizo provided the basis for model creation. First, they were imported directly into Materialise Mimics medical imaging software. Since the segmentation phase incorporated one of every three image slices, the models initially appeared as a series of thin slices with gaps in between them. Using the wrapping feature in Mimics, geometry was added to surround these original models to fill any gaps in the topology.

From Mimics, the wrapped models were exported as .stl files. These .stl files were brought into Autodesk 3ds Studio Max modeling and animation software. The individual objects in $3 \mathrm{ds}$ Max were exported as .obj files. These .obj files were opened in Pixologic ZBrush sculpting software. In ZBrush, each model was refined and any irregularities or artifacts from the segmentation phase were removed. They were then retopologized using the Dynamesh function. This procedure organized the geometry of the models, while preserving their texture and level of detail. After the models were retopologized, they were saved as ZBrush "tools" and exported as new .obj files. These new .obj files were brought back into $3 \mathrm{ds}$ max to replace the original ones.
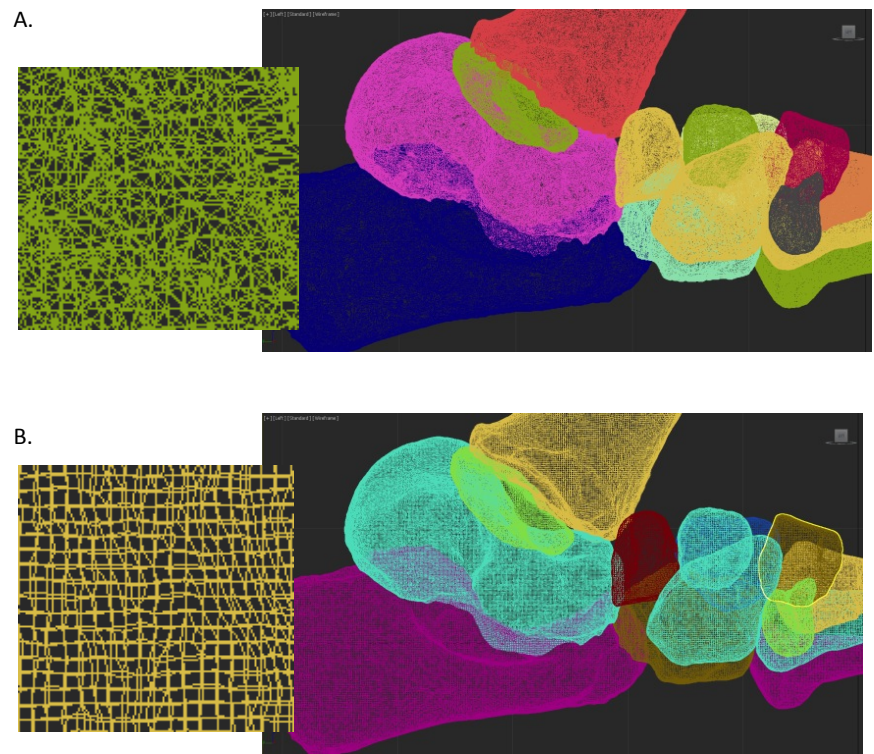

Figure 4. Lateral view of the segmented models in 3ds Max (A) before and (B) after the retopologization process.

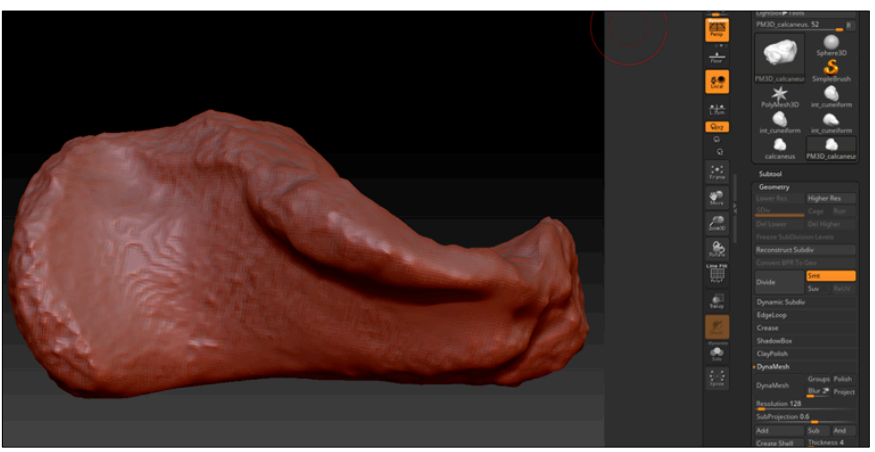

Figure 5. Retopologization interface in Pixologic ZBrush. 
Script: Dr. Alemseged and the rest of the research committee developed a script for the animation. The content was informed largely by the literature on the bipedalism debate and the interpretations of the DIK-1-1 fossil. The script begins with a brief introduction to bipedal locomotion and its uniqueness in the human evolutionary trajectory. It then introduces the DIK-1-1 fossil and explains its importance to the study of human evolution. This section begins with a brief summary of the fossil's discovery and the fossilization process. The narration then focuses on the fossil's foot. This is followed by a discussion of several key features of the foot that have implications for locomotion. The script concludes by describing the overall "functional dichotomy" that defines Selam's skeletal anatomy, according to the existing literature. The script was completed following several rounds of revision and suggestions by the research committee. One major consideration during the script writing process concerned optimizing the experience for the target audience. Viewers in the general population typically attend more to auditory information while viewing an animation (Young 2018). In contrast, viewers with advanced knowledge on the subject, such as paleoanthropologists, tend to focus more on visual information in an animation (Young 2018). Thus, the script was intentionally written at a level that caters to a general audience with basic scientific knowledge, but without specific expertise in the subject.

Storyboard: Because it is helpful to consider visual components of the animation during the development of the audio and narration, the storyboard was created congruously with the script. The introductory sequence depicts the Ethiopian environment with the excavation site and gradually develops into the Late Pliocene landscape. The most current literature on Selam proposes that her upper body was adapted for arboreal locomotion like modern apes; thus, in the 2D section of the animation Selam is shown climbing in an arboreal environment. The screen is partitioned, however, to reveal the remaining half of the narrative. Selam's lower body appears to be adapted for bipedal locomotion like modern humans; thus, this section of the animation also depicts her walking upright in a terrestrial environment. The animation changes settings and enters a 3D environment when the narration focuses on the skeletal anatomy of the foot. This section shows the reconstructed fossil shifting from the distorted and compressed position in which it was discovered, into the original anatomical position.

The fossilized foot skeleton then rotates around a pivot point so that specific relationships and features may be called out. The major features unique to this fossil that can enhance a researchers' understanding of bipedal locomotion are: (a) the metatarsals, which help form the arches of the feet; (b) the medial cuneiform and its articulation with the first metatarsal, which influences the opposability of the hallux; and (c) the articulation of the talus with the calcaneus, which affects the stability of the ankle joint. Visually, the animation was storyboarded so that the introductory and concluding $2 \mathrm{D}$ sections bookended the central, most important 3D section. The animation concludes with a visual recap of the introductory sequence of Selam's different possible locomotor patterns.

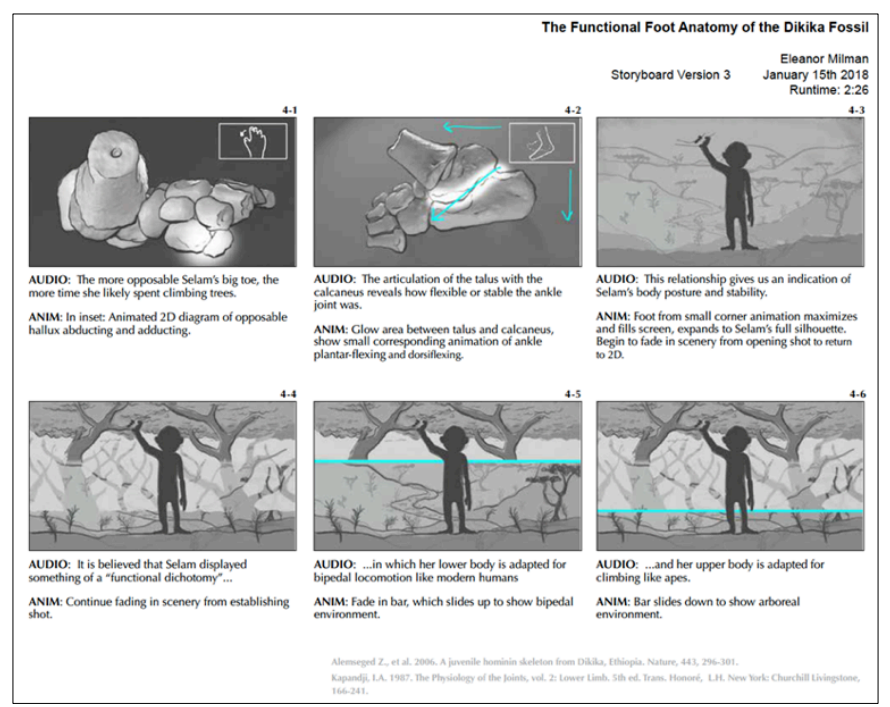

Figure 6. Storyboard page 4 depicting ending transition from 3D into $2 \mathrm{D}$ in the final third of the animation. Blue lines indicate camera transitions and movements.

Animated Storyboard: Upon completion of the script and storyboard, an animated storyboard was created. This was done to test the timing and content of the animation early. The script was read and recorded. The storyboard frames were brought into Adobe After Effects and arranged to correspond with the content of the audio. Camera movements and overall timing of the animation were tested. In the production phases, 2D animated scenes were replaced with original excavation footage. Thus, the original storyboard differs from the final product.

Human CT Data: A significant portion of the animation describes what the Dikika fossil may reveal about human bipedal locomotion, therefore it was necessary to include some comparative anatomy. Originally, it was proposed that Selam be compared with analogous skeletons of both modern humans and extant great apes. However, after a review of the literature on human-ape comparisons within the paleoanthropological community, this plan was revised. Researchers who rely on comparisons between fossils of human ancestry and that of modern apes may risk perpetuating a problematic linear idea of humans' evolutionary trajectory, implying that humanlike implies advanced, and apelike implies primitive (Kimbel and Delezene 2009). The purpose of this research is instead to visually explore what this specific fossil can reveal about human bipedal locomotion and how this locomotor pattern may have manifested in $A$. afarensis in the juvenile life stage. Therefore, only human CT data was used for comparison. 
Because this research explores locomotor patterns in juveniles specifically, the comparison human data needed to come from a child. A DICOM set of CT image data from an eleven-year old male patient was procured from the UIC Department of Orthopaedics. Permission to use the data was granted by Dr. Edward Abraham at UIC's Department of Orthopaedic Surgery. Upon IRB approval to use the data in the animation, the DICOM set was opened in OsiriX medical visualization software. The skeletal anatomy of the foot was isolated from the soft tissues. The whole foot skeleton model was then imported into Pixologic ZBrush. In ZBrush, each individual bone was converted into its own $3 \mathrm{D}$ object as a ZBrush subtool. The geometry of the bones were individually retouched and refined to remove artifacts from OsiriX. Finally, each bone model was exported from ZBrush as an .obj file and imported into 3ds Max.
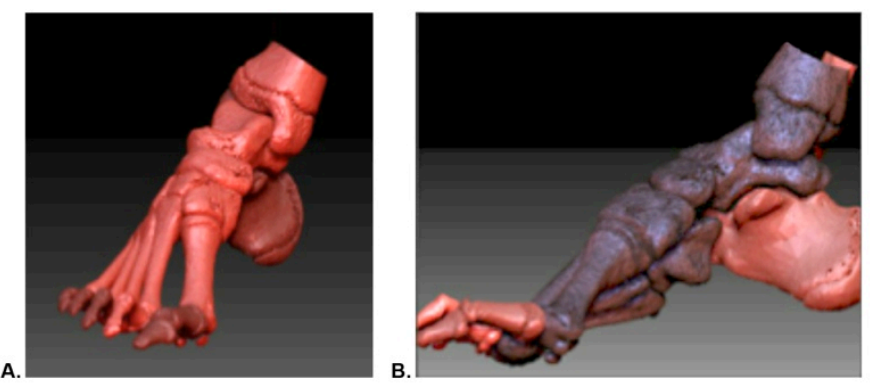

Figure 7. Human foot skeleton undergoing retouching process in Pixologic ZBrush, (A) anteromedial and (B) medial views.
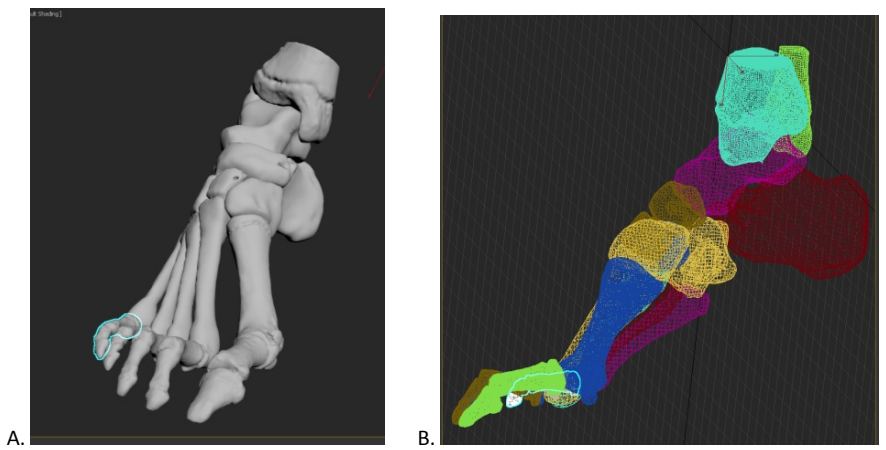

Figure 8. Human foot skeleton in 3ds Max, with (A) surface topology and (B) wireframe shown.

The data from the 11-year-old's CT scan served two purposes: (1) visual comparison with the Dikika fossil, and (2) reference models for the missing skeletal anatomy in the Dikika fossil. The skeletal features of Selam's foot that were not preserved during the fossilization process were substituted with human CT data and visually distinguished from the original fossil. Once imported into 3ds Max, copies of all the metatarsals and phalanges were made. These copies were scaled down to match the size of the Dikika fossil. They were then placed in the position of the missing skeletal features.

\section{Production}

Voice Talent: A voice artist recorded the script in-person
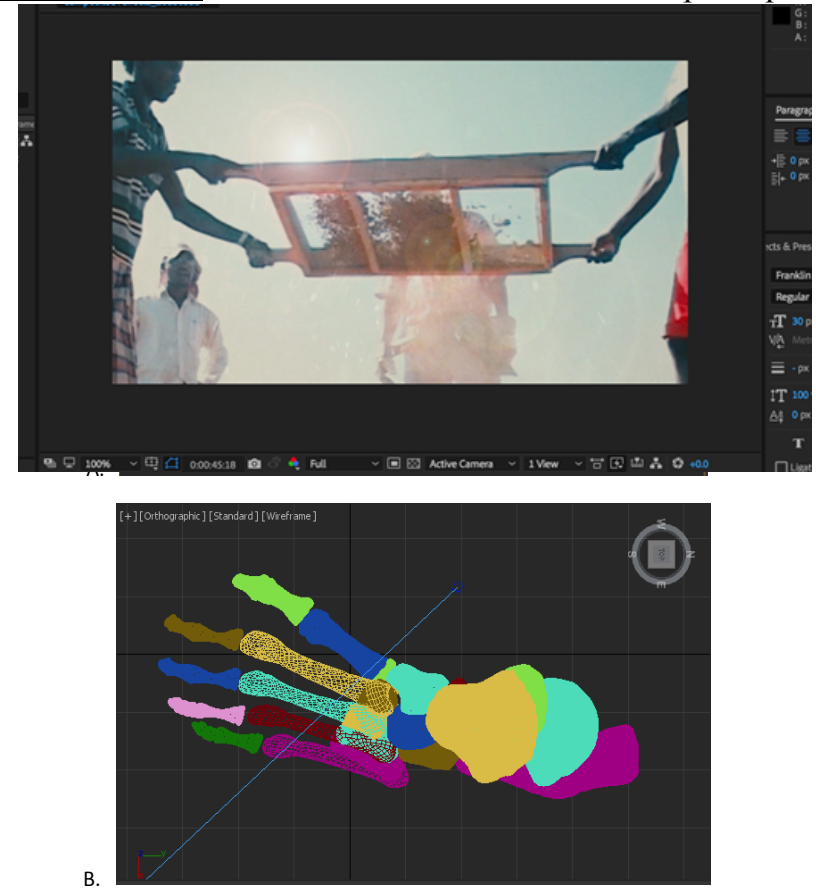

with the supervision of the principal investigator. The voice artist was given the final script in advance of the recording so that she could practice reading it aloud. A professional-grade microphone was used to record, in conjunction with Adobe Audition sound editing software. Two takes of the audio were collected and one was selected to be used in the animation.

Figure 9. Initial placement of human metatarsals and phalanges as placeholders for missing elements of Dikika foot skeleton in (A) medial and (B) superior views.

Footage: Dr. Alemseged provided several minutes of footage from the excavation of the Dikika fossil, courtesy of ZANA Productions/Tefera Ghedamu. This footage was used in the introductory and concluding sections of the animation and was integrated with the 3D sections. In Adobe After Effects, the footage was spliced, restored, and enhanced. The original footage was restored using a series of Red Giant plugins for After Effects. First, the Shooter Suite Frames effect was applied to reduce the interlacing of the footage. Then, the Looks effect was applied to establish a uniform appearance of all the clips. Finally, the Film effect was applied to provide more context to the existing film grain and noise. The clips were then arranged to frame the 3D animation in order to best convey a cohesive story of the Dikika fossil.

Figure 10. Screenshot of footage-editing interface in Adobe After

Effects. This photo depicts the sifting of fossil-bearing material through a screen mesh. 
3D: Materials and Lighting: The retopologized and retouched models were oriented in $3 \mathrm{ds}$ Max. The Redshift rendering engine was selected. A basic lighting setup was created to simulate an outdoor environment. The lighting was intended to maximize the visibility of the surface detail in the fossilized skeleton. A natural, weathered bone material was created for the Dikika fossil. A cleaner natural material was used for the human bones to indicate that it belongs to a modern (vs. fossilized) specimen. In contrast, a plastic material was created for the human bone copies that represented the missing pieces of the Dikika fossil. This provided visual distinction between the original fossil specimen and the reconstructed elements.

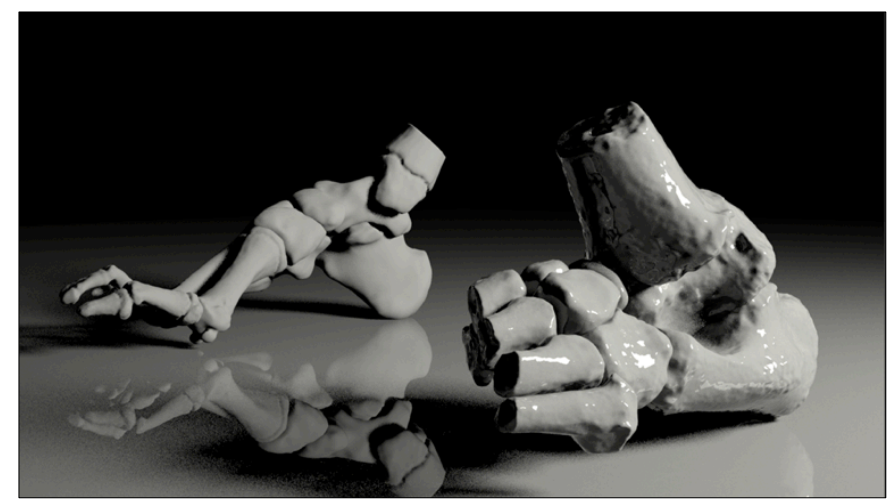

Figure 11. Early test render of lighting setup in 3ds Max.

3D: Animations and Camera Movements: Once the 3D scene was established with the proper lighting and materials, the animation was created. The skeleton of the Dikika foot was aligned into anatomical position from their distorted and compressed orientation. The bones were separated from each other in an exploded view so that the viewer could appreciate their structure and location. The bones were positioned into the orientation that would accompany weight-bearing of the foot and then returned to anatomical position. The hallux, including the reconstructed metatarsal and phalanges, was abducted and adducted to show the role of the medial cuneiform in opposability. The human foot was placed into a separate 3D scene with a copy of the Dikika foot and both were rotated so that the viewer could appreciate major similarities and differences. Cameras were created in the 3D scenes. The movements of the cameras were purposefully subtle to draw the viewer's attention to the animated content.

3D: Rendering: Throughout the modeling, lighting, and animation phases, test renders were created as needed. The Redshift rendering engine was used. Each section was given its own $3 \mathrm{ds}$ Max scene with render settings that optimized render time and image quality. Multiple arbitrary output values (AOVs), also known as render passes, were created: beauty (the original image), shadows, object IDs, subsurface scattering, and reflections. All sections of footage contained an image for each the AOVs. The footage was sent to the render farm in the Applied Health Sciences building at UIC.

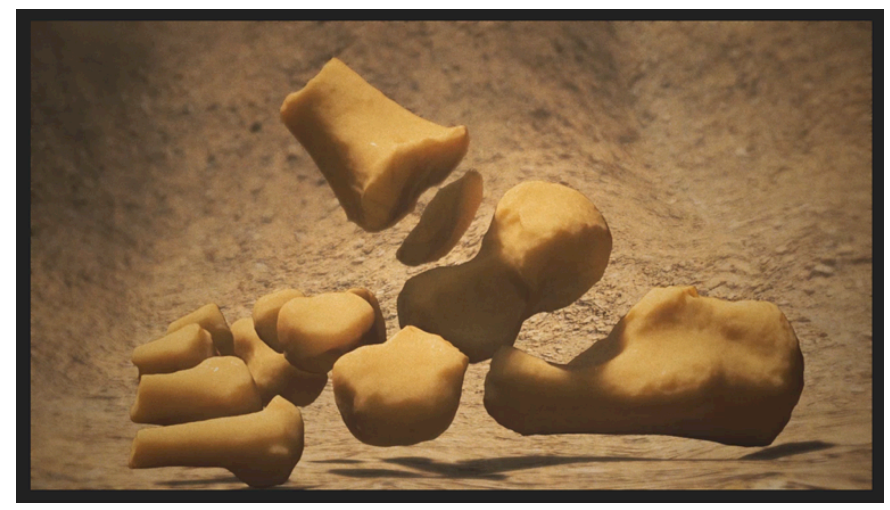

Figure 12. Screen capture of exploded Dikika foot skeleton.

\section{Postproduction}

Compositing: Each scene, including all its AOVs, was imported as a composition into After Effects. The beauty pass was placed at the bottom of the layer stack, followed by the shadows, subsurface scattering, and reflections. Object IDs were placed on top and a color key was used to isolate different individual bones as needed. Blending modes between the composite layers were adjusted to create the desired realism in the animation. In total, five After Effects compositions were created to complete the compositing process to optimize workflow and buffering time.

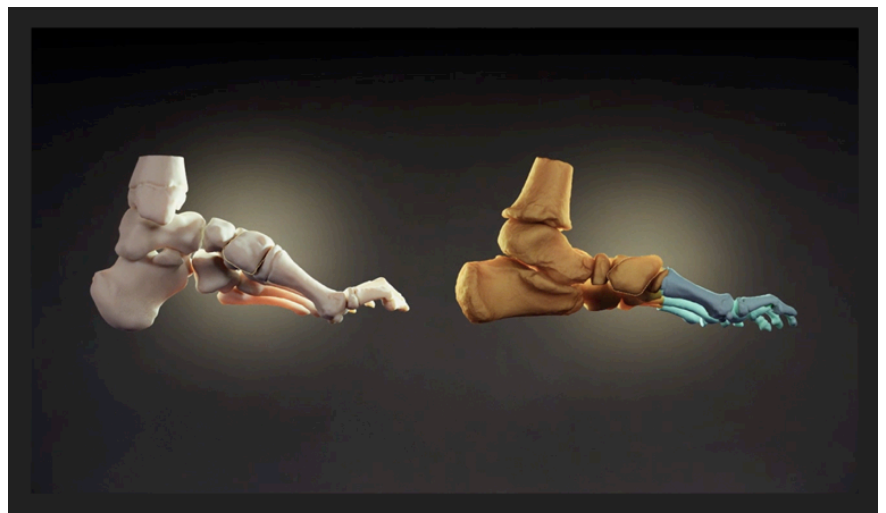

Figure 13. Screen capture of composited scene with comparison between the human (left) and Dikika foot (right) skeletons.

Sound Editing: The narrated script was imported into Adobe Audition for final editing. Extra- environmental noise was eliminated, and the audio file was trimmed to suit the length of the animation. Open-source music (written and performed by the Akoko Nante Ensemble, sourced at freemusicarchive.org) was downloaded and imported into After Effects to accompany the animation. The final voiceover file was also imported. Final timing adjustments were made with the footage.

Final Editing: The opening title sequence, closing credits, labels, and other graphic elements were all added and animated in After Effects. This helped unify the look and feel of the $2 \mathrm{D}$ and $3 \mathrm{D}$ sections of the animation. Finally, the 
animation was rendered from After Effects using Adobe Media Encoder as an mp4 file.

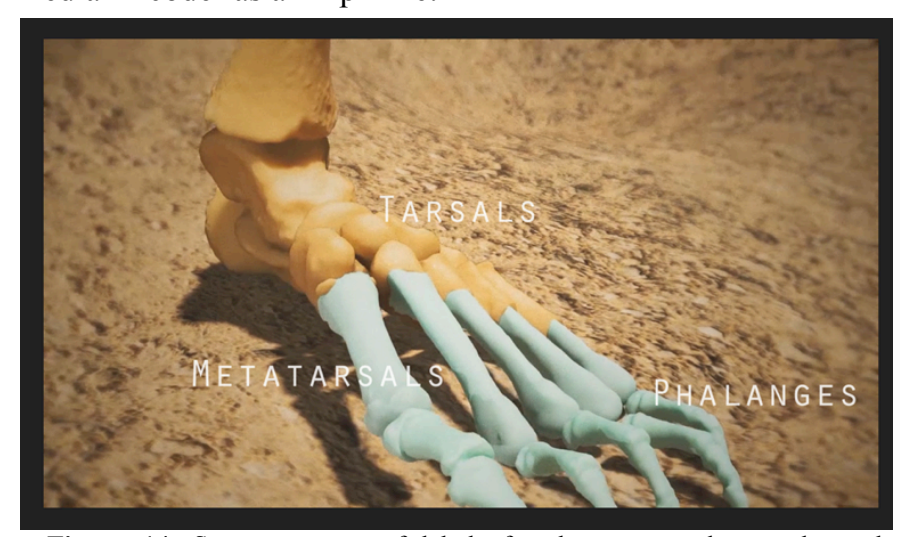

Figure 14. Screen capture of labels for the metatarsals, tarsals, and phalanges of the Dikika foot skeleton.

\section{Evaluation Plan}

The final product was qualitatively evaluated by Dr. Alemseged and the rest of the research committee. This evaluation assessed the animation's ability to facilitate visualization of existing literature on the functional anatomy of the Dikika foot and communicate the taphonomy and overall story of this fossil. Feedback from the research committee focused on the success with which the animation enables further paleoanthropological investigation of mechanical function. The animation's ability to facilitate public outreach and contribute to the study of human evolution was also considered. At each major checkpoint throughout the preproduction, production, and postproduction phases, the project was reviewed by the research committee and revised as needed.

\section{Results}

The major goals of this animation were to (1) highlight Selam's functional foot anatomy and compare it with that of Homo, and (2) depict the narrative of the fossilization process and the significance of this particular specimen. A final committee meeting was arranged to determine the success with which the project accomplished the above goals. This qualitative review of the overall project concluded that the animation, and the process used to create it, did indeed achieve the intended purpose. Thus, the original research question can be answered: biomedical visualization techniques can be employed in the development of an animation that depicts the history and existing literature on the functional foot anatomy of DIK-1-1. The animation presents the story and context of this influential discovery and enhances the ability of scientists to communicate their research to various audiences.

\section{Discussion}

\section{Review of Major Points}

The evolutionary origin of human bipedal locomotion is a complex and highly debated topic in paleoanthropological research. Australopithecus afarensis is one of the earliest hominins to a display humanlike walking pattern, which makes it a crucial species in the study of locomotion. Much of this research focuses on the skeletal morphology of the lower limb, especially the foot. In the past, analyses of fossilized specimens have polarized researchers as to how A. afarensis moved about its environment three million years ago (Stern 2000). To date, a consensus has yet to be reached about this moment in human evolutionary history.

The discovery of DIK-1-1 allows paleoanthropologists to examine the locomotion of $A$. afarensis in a juvenile individual, which can provide new insight about its developmental trajectory. With modern techniques such as 3D modeling and animation, it is possible to visually present the paleoanthropological context of DIK-1-1 and the existing research on the functional anatomy of this individual. With this project, researchers may be able to examine and test their own hypotheses and visually evaluate existing theories about locomotion, A. afarensis, and even modern humans.

\section{Limitations}

The data used for this project possessed several intrinsically limiting factors. The Dikika fossil was scanned using CT; thus, each image slice needed to be segmented by hand. This involved some interpretation of the data in attributing specific zones of the images to either rock matrix or fossilized bone. To mitigate this limitation, Dr. Alemseged and his lab manager were present during this process and were consulted thoroughly at each moment where a paleoanthropologist's expertise was required. While the ankle bones were preserved and their implications for functional anatomy could be analyzed, parts of the metatarsals and phalanges were not preserved and needed to be reconstructed using the available $\mathrm{CT}$ data of a juvenile modern human.

The human skeletal data that was used as a comparison also came with several limitations. First, Selam was three years old when she died; the human specimen in the animation was eleven years old. It is important to acknowledge that the age discrepancy between these two specimens influences the extent to which they may be used as homologs. However, $A$. afarensis likely experienced a more rapid life history and developmental trajectory than modern humans. Thus, it is possible that these specimens are more homologous than they would be if the animation were comparing specimens of the same species. Second, the human specimen displays some inversion in the skeletal anatomy of the foot. This was corrected once the 3D models of each individual foot bone were brought into 3ds Max.

A modest level of interpretation was involved in placing the Dikika foot into anatomical position. Because this fossil is the youngest early hominin specimen available, limited visual references exist for its exact orientation in vivo. The 
references used included the juvenile human foot and the adult $A$. afarensis foot. A similar limitation was encountered when scaling the human foot relative to the Dikika foot.

\section{Implications for the Profession}

This research benefits the field of Biomedical Visualization on several grounds. First, Avizo software is currently seldom used to create models that are animated in $3 \mathrm{ds}$ Max. The use of Avizo, which is often applied in investigations of nonliving specimens, helps expand biomedical animators' technology toolkit for science visualization. To the best of the investigator's knowledge, the workflow from Avizo to Mimics to 3ds Max had not previously been attempted.

The blended use of video footage and 3D animation with simple narration helps expand the audience that may learn from this animation. The overall story of the fossil presented in $2 \mathrm{D}$ and the intricacies of the Dikika foot structure in 3D may provide a useful framework in future animations of early hominin fossil reconstruction.

Further, an important relationship was established between the Biomedical Visualization department at UIC and the Alemseged $\mathrm{Lab}$ at $\mathrm{U}$ of $\mathrm{C}$. The strength of visualizations of human evolution and physical anthropology relies strongly on the interdisciplinary collaborations between paleoanthropologists and visualization specialists. As the impact of this project is determined, other collaborations may be possible and more paleoanthropological visualizations may be made for researchers and a general audience.

\section{Future Applications}

This research is not intended to propose claims about the skeletal morphology of the Dikika Child or the way she may have moved about her environment. Rather, it presents the existing literature on this fossil and visualizes it in an accessible way. This allows researchers and the public to engage with revolutionary paleoanthropological research more easily than is possible with the existing visual resources.

As research on the Dikika Child continues and the knowledge on bipedal locomotion is refined, this animation may be used to augment the existing literature for both researchers developing hypotheses and the public.

\section{Acknowledgements}

The authors wish to thank Kelsi Hurdle, Dr. Alemseged's lab manager. Thank you to Susan King and Dr. Edward Abraham of for permission to use patient data from UIC Orthopaedics. Thank you to the excavation team who discovered and preserved the Dikika fossil.

\section{References}

Alemseged Z., Spoor, F., Kimbel, W.H., Bobe, R., Geraads, D., Reed, D., and Wynn, J.G. (2006). A juvenile early hominin skeleton from Dikika, Ethiopia. Nature, 443, 296-301.

Boucher, E. M., and Artz, T. (2013, Feb. \& March). Digital paleoart: reconstruction and restoration from laser-scanned fossils (Doctoral dissertation).

Cunningham, J.A., Rahman, I.A., Lautenschlager, S., Rayfield, E.J., and Donoghue, P.C.J. (2014). A virtual world of paleontology. Trends in Ecology \& Evolution, 29(6), 347-57.

Duncan, A.S., Kappelman, J., and Shapiro, L.J. (1994). Metatarsophalangeal joint function and positional behavior in Australopithecus afarensis. American Journal of Physical Anthropology, 93, 67-81.

Gill, C.M., Bredella, M.A., and DeSilva, J.M. (2015). Skeletal development of hallucal tarsometatarsal joint curvature and angulation in extant apes and modern humans. Journal of Human Evolution, 88, 137-145.

Green, D.J., and Alemseged, Z. (2012). Australopithecus afarensis scapular ontogeny, function, and the role of climbing in human evolution. Science, 338, 514-517.

Harcourt-Smith, W.E.H., and Aiello, L.C. (2004). Review: Fossils, feet and the evolution of human bipedal locomotion. Journal of Anatomy, 204, 403-416.

Henning, K. (2012). Visualization and stature estimation of a mid-Holocene male skeleton. Masters project research. University of Illinois at Chicago.

Kanamoto, S., Ogihara, N., and Nakatsukasa, M. (2011). Three-dimensional orientations of talar articular surfaces in humans and great apes. Primates, 52, 61-68.

Kimbel, W.H. and Delezene, L.K. (2009). Lucy redux: A review of research on A. afarensis. Yearbook of Physical Anthropology, 52, 2-48.

Münzer, S. (2014). Facilitating recognition of spatial structures through animation and the role of mental rotation ability. Learning and Individual Differences, 38, 76-82.

Padilla, A. (2015). Non-invasive digital excavation and threedimensional reconstruction of human paleontological remains. Masters project research. University of Illinois at Chicago.

Sisk, M. (2011). A facial reconstruction and animation of an early Holocene male utilizing traditional facial reconstruction methods, age-progression techniques and 3D computer graphic visualization. Masters project research. University of Illinois at Chicago. 
Spoor, F., Jeffery, N., and Zonneveld, F. (1999). Review: Using diagnostic radiology in human evolutionary studies. Journal of Anatomy, 197 61-76.

Spoor, F., Jeffery, N., and Zonneveld, F. Chapter 6: "Imaging skeletal growth and evolution" in Development, Growth and Evolution. (2000). The Linnean Society of London, 124-161.

Stern, J.T. JR. (2000). Climbing to the top: A personal memoir of Australopithecus afarensis. Evolutionary Anthropology, 113-133.

Young, C., Associate Clinical Faculty at University of Illinois at Chicago. (2018). (Personal correspondence).

Zumach, K. (2017). Documenting Visualization Methodology and Decision-Making in Digital Reconstruction of Norellius nyctisaurop. Masters project research. University of Illinois at Chicago.

\section{Authors}

Eleanor Milman, BA, MSc, is a Chicago-based medical illustrator and animator specializing in online patient education. She obtained her Bachelor of Arts in Psychology at McGill University in Montreal, QC and her Master of Science in Biomedical Visualization at the University of Illinois at Chicago. Her other professional interests include applied bioethics, physical anthropology, and neuropsychology.

Email: eleanor.milman@gmail.com

John M. Daugherty, MS, CMI, FAMI, is program director of the UIC Biomedical Visualization graduate program. He is the recipient of numerous honors and awards for his illustration work in medical education and healthcare marketing, including over twenty salon awards and several Ralph Sweet Member's Choice Awards (Best of Show) from the Association of Medical Illustrators (AMI). He is a fivetime recipient of the Excalibur Award for Teaching Excellence from the UIC AHS Student Council, a recipient of the Emil W. Hospodar Excellence in Teaching Award from UIC in 2008, and a recipient of the Brödel Award of Excellence in Education from the AMI in 2013.

Zeresenay Alemseged, $\mathbf{P h D}$, is the Donald N. Pritzker Professor at the University of Chicago where he teaches and researchers on the biological and cultural origins of our species. He is an Ethiopian Paleo-Anthropologist and got his $\mathrm{PhD}$ from the University of Sorbonne in Paris in 1998. Following that he worked as a Postdoctoral Fellow at Arizona State University (2000 -2003), a Senior Scientist at the Max Planck Institute (Germany 2004 - 2008) and a Senior Curator of Anthropology at the California Academy of Sciences (2008 - 2016). He has published over 60 peer reviewed research papers in Nature, Science, the Proceeding of the National Academy of Sciences and other specialized journals and books. He also regularly engages the public and has been featured on CNN, BBC, National Geographic and TED talks. He developed the popular exhibit on human evolution known as "The Human Odyssey" currently on display at the California Academy of Science. Among many honors he is a Fellow of the American Association for the Advancement of Science and Explorer for National Geography.

Kevin Brennan, M.S., CMI, was the Senior Content Developer and Medical Animator at Visible Body and more recently was an Assistant Clinical Professor at the University of Illinois at Chicago. He is the founder of BioViz Studios, a small medical animation company in Chicago's Medical District.

Leah Lebowicz, MS, CMI, is a Clinical Assistant Professor and Associate Program Director of the Biomedical Visualization Graduate program (BVIS) at the University of Illinois at Chicago. As part of the BVIS faculty, Leah teaches the 3D modeling courses and Visual Learning and Thinking II courses in the program. She is interested in improving medical education with the use of $3 \mathrm{D}$ interactive programs, specifically in the field of embryology. Leah has served as a research chair and committee member on numerous Master student research projects since 2012. The research topics she and her students have investigated have involved 3D modeling, 2D and 3D animation, interactive program design and development, virtual reality, embryology, medical education, paleontology reconstructions, and visualizing medical diagnostic procedures.

\section{Licensing}

The authors have chosen to license this content under a Creative Commons Attribution, NonCommercial, NoDerivatives 4.0 International License.

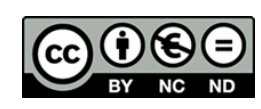

